\title{
Enhanced Optical Cross Section via Collective Coupling of Atomic Dipoles in a 2D Array
}

\author{
Robert J. Bettles, ${ }^{*}$ Simon A. Gardiner, ${ }^{\dagger}$ and Charles S. Adams ${ }^{\ddagger}$ \\ Department of Physics, Joint Quantum Center (JQC) Durham-Newcastle, Durham University, \\ South Road, Durham DHI 3LE, United Kingdom \\ (Received 27 October 2015; published 9 March 2016)
}

\begin{abstract}
Enhancing the optical cross section is an enticing goal in light-matter interactions, due to its fundamental role in quantum and nonlinear optics. Here, we show how dipolar interactions can suppress off-axis scattering in a two-dimensional atomic array, leading to a subradiant collective mode where the optical cross section is enhanced by almost an order of magnitude. As a consequence, it is possible to attain an optical depth which implies high-fidelity extinction, from a monolayer. Using realistic experimental parameters, we also model how lattice vacancies and the atomic trapping depth affect the transmission, concluding that such high extinction should be possible, using current experimental techniques.
\end{abstract}

DOI: 10.1103/PhysRevLett.116.103602

Strong coupling between light and matter has been a long sought-after goal. Light-matter coupling can be conveniently characterized in terms of extinction which corresponds to the probability for a medium to remove a photon from an incident field. For a single dipole, the highest recorded extinctions, of order $10 \%$, have been achieved using individual molecules [1] and atoms [2,3]; single dipole extinction has also been demonstrated using ions [4] and quantum dots [5]. The free-space extinction is typically limited by the focusing strength of a lens or mirror [2] and can be further enhanced using a waveguide or cavity thereby attaining the so-called strong coupling regime associated with cavity QED [6,7]. Replacing the single dipole with a high density ensemble of dipoles can have a dramatic effect on the optical response [8]. Coherent scattering between dipoles results in collective behavior, which can include enhanced or reduced scattering rates (superradiance or subradiance, respectively) [9-12], line shifts [11,13,14], and interference line shapes [15-17]. Recent experiments have shown that at high densities the dipole-dipole interaction in random atomic ensembles can significantly attenuate the optical extinction in both very hot $(\sim 100 \mathrm{~K})$ [18] and cold [19-21] $(\sim 100 \mu \mathrm{K})$ atomic vapors. Placing scatterers in a regular array formation can further enhance the cooperative response. Examples include near perfect extinction and transmission through arrays of gold nanorods [22], linewidth narrowing in metamolecules [23], and extraordinary optical transmission in hole arrays [24]. In addition to diffraction and interference effects, the coupling to collective and plasmonic modes plays a crucial role in explaining these phenomena [25-27]. Cooperative broadening and shifts $[15,28]$ as well

Published by the American Physical Society under the terms of the Creative Commons Attribution 3.0 License. Further distribution of this work must maintain attribution to the author $(s)$ and the published article's title, journal citation, and DOI. as subwavelength excitation [17] have been predicted in analogous atomic dipolar arrays, with the advantages that atomic systems allow easy access to the quantum regime, have much higher $Q$ factors, and significantly less nonradiative decay than the aforementioned plasmonic systems. In this Letter, we show that atomic $2 \mathrm{D}$ arrays can also exhibit extreme variation in transmission depending on geometry. For certain magic lattice spacings, high-fidelity extinction can occur, corresponding to an enhanced atomlight coupling which may open the door to exciting new applications in quantum simulation and information processing. Unlike the photonic band gaps predicted in $3 \mathrm{D}$ atomic lattices [29,30], extinction in our system is due to a subradiant mode rather than a gap in the density of states.

Extinction, like many light-matter phenomena, is an interference effect. The total electric field at position $\mathbf{r}$, $\mathbf{E}(\mathbf{r})=\mathbf{E}_{0}(\mathbf{r})+\sum_{i} \mathbf{E}_{i}(\mathbf{r})$, is the sum of the driving field, $\mathbf{E}_{0}(\mathbf{r})$, and the fields radiated by the $N$ scatterers, $\sum_{i=1}^{N} \mathbf{E}_{i}(\mathbf{r})$; extinction of the driving field occurs when the driving and scattered fields interfere destructively. The scattered field from an electric dipole $\mathbf{d}_{i}$ located at $\mathbf{r}_{i}$ is $\mathbf{E}_{i}(\mathbf{r})=\mathrm{G}\left(\mathbf{R}_{i}\right) \mathbf{d}_{i}$, where $\mathrm{G}\left(\mathbf{R}_{i}\right)$ is the dipole propagation tensor [Eq. (S1) in the Supplemental Material [31]] and $\mathbf{R}_{i}=\mathbf{r}-\mathbf{r}_{i}$. This dipole moment, in turn, is driven by the total local electric field, $\mathbf{d}_{i}=\alpha \mathbf{E}\left(\mathbf{r}_{i}\right)$, where $\alpha$ is the dipole polarizability. For a closed two-level $J=0 \rightarrow J=1$ atomic transition (e.g., Sr [34] or Yb [35]), the polarizability takes the form $\alpha=-\alpha_{0} /\left[\left(\Delta / \gamma_{0}\right)+\mathrm{i}\right]$ where $\alpha_{0}=6 \pi \varepsilon_{0} / k_{0}^{3}, \varepsilon_{0}$ is the permittivity of free space, $\lambda_{0}=$ $2 \pi / k_{0}$ is the wavelength of the dipole transition, $2 \gamma_{0}$ is the excited state decay rate, and $\Delta=\omega-\omega_{0}$ is the detuning of the driving frequency $\omega$ from the transition frequency $\omega_{0}$. A similar treatment can applied to plasmonic nanoresonators [36,37]. The linear response of $\mathbf{d}_{i}$ to $\mathbf{E}$ implies weak driving and means our model is closely equivalent to a set of damped driven classical oscillators [38]. The weak 
driving limit can, nonetheless, be used to predict the extinction occurring in the quantum limit. Optimizing this extinction involves matching the spatial $[2,39,40]$ and temporal [41] modes of the incident field to the field scattered by the dipoles. The scattered field of a single dipole has a very similar spatial mode profile to a Gaussian beam tightly focused on that dipole [31]. The difference between the two fields along the axis of propagation of the Gaussian beam is simply a numerical factor $k^{2} w_{0}^{2} / 3$ where $k=2 \pi / \lambda$ is the beam wave number (we assume the rotating wave approximation and, hence, $k \simeq k_{0}$ ), and the beam waist $w_{0}$ is the $1 / e$ radius at the focus. Maximizing the overlap would require a tightly focused beam $[2,39]$ (using, e.g., a high numerical aperture lens) with waist of order $w_{0} \simeq 0.3 \lambda$-far beyond the reach of conventional free-space lenses. The alternative we propose in this Letter is to replace the single dipole with a monolayer of dipoles, which can exhibit near $100 \%$ extinction without the need for such strong focusing. If combined with Rydberg blockade, this could be employed to realize a high-fidelity photonic gate [42].

The case of many dipoles is less trivial than for a single dipole, since now the local field experienced by each dipole is both the external driving field and also the fields scattered by the other $N-1$ dipoles, $\mathbf{E}\left(\mathbf{r}_{i}\right)=\mathbf{E}_{0}\left(\mathbf{r}_{i}\right)+\sum_{j \neq i} \mathbf{E}_{j}\left(\mathbf{r}_{i}\right)$. For an inhomogeneously broadened ensemble (e.g., a hightemperature thermal vapor [13]), the sum of scattered fields $\sum_{j \neq i} \mathbf{E}_{j}\left(\mathbf{r}_{i}\right)$ can be replaced by an ensemble averaged mean field, resulting in, e.g., a geometry-dependent cooperative Lamb shift $[13,43,44]$. The case we are interested in here is the homogeneously broadened regime (where atomic motion can be ignored $[15,45])$, for which the recurrent scattering between dipoles must be included [44]. Substituting $\mathbf{d}_{i}=\alpha \mathbf{E}\left(\mathbf{r}_{i}\right)$ into the equation for the local fields results in a set of coupled linear equations,

$$
\mathbf{d}_{i}=\alpha\left(\mathbf{E}_{0}\left(\mathbf{r}_{i}\right)+\sum_{j \neq i} \mathbf{G}\left(\mathbf{R}_{i j}\right) \mathbf{d}_{j}\right),
$$

where $\mathbf{R}_{i j}=\mathbf{r}_{i}-\mathbf{r}_{j}$. These can be solved numerically for modest $N$ with arbitrary dipole positions and driving fields [15,45-47].

To measure transmission and extinction, we calculate the total power passing through a lens downstream of the dipolar ensemble. The power is related to the Poynting vector,

$$
P=\frac{\varepsilon_{0} c^{2}}{2} \int_{L} \Re\left[\mathbf{E} \times \mathbf{B}^{*}\right] \cdot d \mathbf{A},
$$

where $c$ is the speed of light, $\mathbf{B}=\hat{\mathbf{k}} \times \mathbf{E} / c$ is the $B$ field for an $E$ field with propagation unit vector $\hat{\mathbf{k}}$, and $d \mathbf{A}=d A \hat{\mathbf{z}}$ is the lens differential area element. We place the lens at $z_{L}=$ $150 \lambda$ centered on $x, y=0$. The lens radius $R_{L}=90 \lambda$ is large enough to avoid finite size effects [31] while having a realistic numerical aperture $\left(\mathrm{NA}=R_{L} / z_{L}=0.6\right)$. The driving field incident on the focusing lens has circular polarization vector $\hat{\boldsymbol{\epsilon}}_{+}=(\hat{x}+i \hat{y}) / \sqrt{2}$. Strong focusing introduces small contributions from $\hat{\boldsymbol{\epsilon}}_{-}=(\hat{x}-i \hat{y}) / \sqrt{2}$ and $\hat{\boldsymbol{\epsilon}}_{z}$, which we account for $[2,31]$. The excited states $m_{J}=\{0, \pm 1\}$ are treated as degenerate; however, driving a closed $m_{J} \leftrightarrow m_{J}+1$ transition gives quantitatively similar values for the optimal extinction. We define transmission as the ratio of the power through the lens in the presence $(P)$ and absence $\left(P_{0}\right)$ of the dipoles, $T=P / P_{0}=e^{-\sigma N_{2 \mathrm{D}} \text {, }}$ where $\sigma$ is the extinction cross section, and $N_{2 \mathrm{D}}$ is the 2D number density. Extinction is defined as $\epsilon \equiv 1-T$. For low densities ( $N_{2 \mathrm{D}} \ll \lambda_{0}^{-2}$, or $N_{3 \mathrm{D}} \ll \lambda_{0}^{-3}$ in 3D ensembles), the local field at each dipole is dominated by the external driving field since the scattered fields from neighboring dipoles in the far field decay with $1 /\left(k_{0} R_{i j}\right)$, where $R_{i j}=\left|\mathbf{R}_{i j}\right|$. In this case, the total extinction cross section is simply the cross section of an independent two-level atom, $\sigma_{\text {ind }}=\sigma_{0} /\left[1+\left(\Delta / \gamma_{0}\right)^{2}\right]$, where $\sigma_{0}=3 \lambda_{0}^{2} /(2 \pi)$. As mentioned in the introduction, recent experiments in dense $\left(N_{3 \mathrm{D}} \gg \lambda_{0}^{-3}\right)$ atomic vapors [18-21] have shown that dipole-dipole interactions reduce the cross section below the noninteracting value $\left(\sigma<\sigma_{\text {ind }}\right)$, increasing the transparency of the medium.

As displayed in Fig. 1, we start by considering resonant $(\Delta=0)$ transmission through a 2D monolayer of uniformly randomly distributed atoms. The black dotted line plots the predicted transmission when ignoring dipole-dipole interactions, corresponding to the $2 \mathrm{D}$ limit of the familiar BeerLambert law $T_{\text {ind }}=\exp \left(-\sigma_{\text {ind }} N_{2 \mathrm{D}}\right)$. In agreement with experiment [18-21], the transmission increasingly deviates

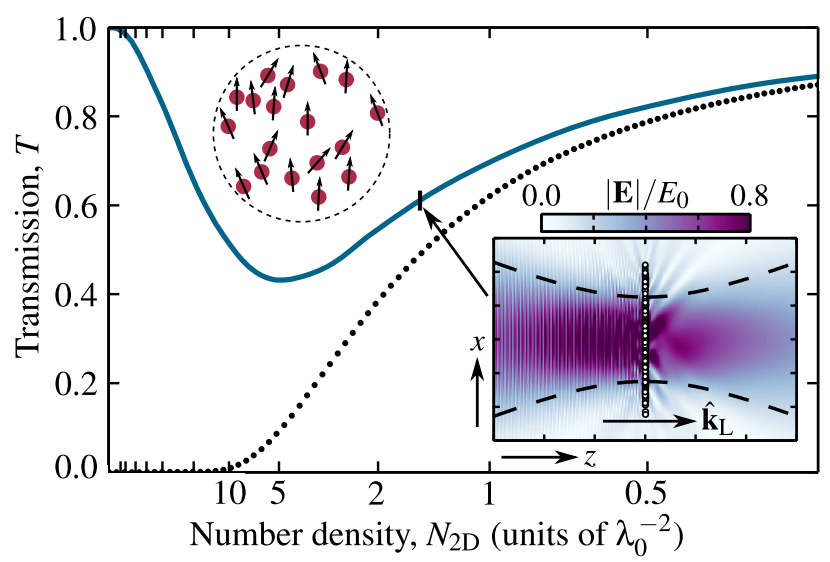

FIG. 1. Resonant optical transmission of a Gaussian beam through a random 2D monolayer of $N=100$ interacting dipoles. As the 2D number density $N_{2 \mathrm{D}}$ increases, the interacting monolayer (blue solid line) deviates from $T_{\text {ind }}$ (black dotted line), which assumes each dipole is a noninteracting opaque disk of cross sectional area $\sigma_{0}$. Each data point is averaged over 100 realizations. The beam waist is $w_{0} \simeq 2.5 \lambda$, and the collection lens has radius $R_{L}=90 \lambda_{0}$ and position $z_{L}=150 \lambda_{0}$. (Inset) Weak cancellation of the total electric field magnitude $|\mathbf{E}|$ in the $x z$ plane downstream of the monolayer $\left(N_{2 \mathrm{D}} \simeq 1.5 \lambda_{0}^{-2}\right)$. $x$ and $z$ vary between $\pm 6 \lambda_{0}$ and $\pm 30 \lambda_{0}$, respectively. The Gaussian beam propagates with vector $\hat{\mathbf{k}}_{L}=\hat{\mathbf{z}}$. The black dashed line shows the $1 / e$ beamwidth and the white circles the atom positions. 
from the noninteracting Beer-Lambert value as the density increases. Shifts diverging as $1 / R_{i j}^{3}$ between closely spaced dipoles result in a broadening and weakening of the overall cross section line shape, reducing the resonant extinction (increasing transmission). It might, therefore, seem that interactions make the extinction worse. However, if we introduce spatial ordering to the atoms by confining them to a fixed regular (triangular) array, with one atom per site, we see in Fig. 2 that the transmission can be significantly lower than both the noninteracting and randomly distributed cases. Such an array could be realized in, e.g., an optical lattice in the Mott-insulator phase [48,49] or spatial light modulator dipole trap array [50]. For a particular magic lattice spacing $\left(a=0.87 \lambda_{0}\right)$, the extinction $(1-T)$ is greater than $99 \%$, corresponding to almost an order of magnitude increase in cross section $\left(\sigma \simeq 7 \sigma_{0}\right)$. Limits on the scattering cross section were discussed in [51]. The efficient cancellation of the electric fields downstream of the lattice can be seen in the inset of Fig. 2, which is contrasted with the poorer extinction and significant scattering out of the beam in the random monolayer (inset, Fig. 1). The transmission minimum also corresponds to a reflection maximum observable in the inset of Fig. 2, as well as by calculating the power reflected back through the focusing lens at $z=-z_{L}$ (reflection $R \gg 98 \%$ ). By slightly changing the lattice spacing $\left(a=0.87 \lambda_{0} \rightarrow 1.05 \lambda_{0}\right)$, the transmission increases from $<1 \%$ to $\simeq 90 \%$. Consequently, the monolayer can be switched between distinct transmission and reflection states, in the same spatial mode, which is the ideal starting point for a gate or all-optical transistor.

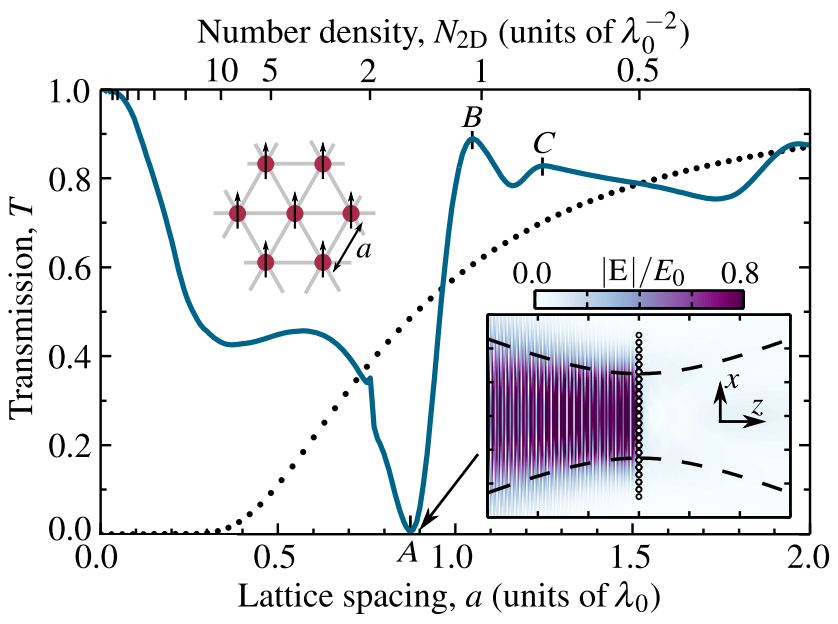

FIG. 2. Resonant optical transmission of a Gaussian beam through a triangular 2D array of $N=102$ interacting dipoles (blue solid line). Unlike the random monolayer in Fig. 1, the transmission goes below and above $T_{\text {ind }}$ (black dotted line). $A, B$, and $C$ correspond to the lattice spacings used in Fig. 3. (Inset) At $a=0.87 \lambda_{0}\left(N_{2 \mathrm{D}} \simeq 1.5 \lambda_{0}^{-2}\right)$, the dipole and driving fields almost perfectly cancel downstream of the lattice, resulting in less than $1 \%$ transmission over the collection lens. The same parameters for the beam, lens, and inset are used as in Fig. 1.
We now address why there is a magic spacing that produces optimal extinction. In Fig. 3, we plot the transmission as a function of detuning at the points labeled $A, B$, and $C$ in Fig. 2. The behavior of the interacting line shapes (blue solid lines) is determined by the eigenmodes of Eq. (1). Each eigenmode contributes a shift $\Delta_{l}$ and linewidth $\gamma_{l}$ proportional to the real and imaginary parts of its eigenvalue, respectively [15,37]. The transmission behavior in Fig. 2 corresponds to the value of the transmission at $\Delta=0$, indicated by the vertical dashed lines in Fig. 3. In Fig. 3(a) the line shape is dominated by two nearly degenerate modes with half-widths $\gamma_{l}=0.37 \gamma_{0}$ centered at $\Delta_{l} \simeq 0$. Extinction cross section scales inversely with linewidth, so subradiance $\left(\gamma<\gamma_{0}\right)$ results in an enhanced extinction. This combined with the maximal extinction at $\Delta=0$, results in the transmission minimum at $a=0.87 \lambda_{0}$ (point $A$ in Fig. 2). By changing the detuning of the driving field, however, we can select a range of spacings over which large extinction is still possible $\left[\epsilon>98 \%\right.$ for $0.67<a / \lambda_{0}<0.92$; see the inset in Fig. 3(a)]. Figures 3(b) and 3(c) correspond to the local transmission maxima at points $B$ and $C$ in Fig. 2. While the peak extinction in Fig. 3(b) is still around 80\%, it is shifted off resonance, so the extinction at $\Delta=0$ is small. In Fig. 3(c), the line shape is centered on $\Delta=0$, although it is now superradiant $\left(\gamma \simeq 2 \gamma_{0}\right)$, and so the peak extinction is reduced.

Large peak extinctions on resonance $(\Delta=0)$ are also possible in square $\left(\epsilon>98 \%\right.$ at $a=0.79 \lambda_{0}$; Fig. 4) and

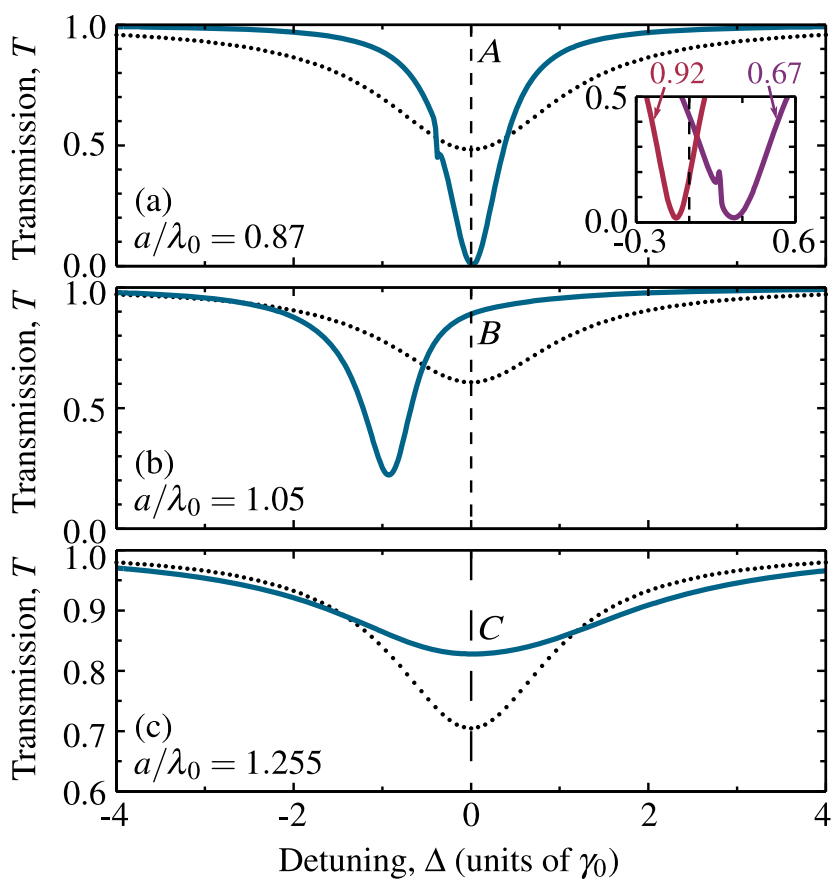

FIG. 3. Transmission as a function of detuning through an $N=102$ triangular lattice of interacting dipoles. The lattice spacings in (a)-(c) correspond to those labeled $A, B$, and $C$ in Fig. 2 (inset: $a / \lambda_{0}=0.67,0.92$ ). The solid lines plot the full interacting transmission for the same beam and lenses as Fig. 1. The dotted lines show $T_{\text {ind }}$ (i.e., assuming no interactions). The vertical dashed lines at $\Delta=0$ have dash lengths $\Delta T=0.05$. 


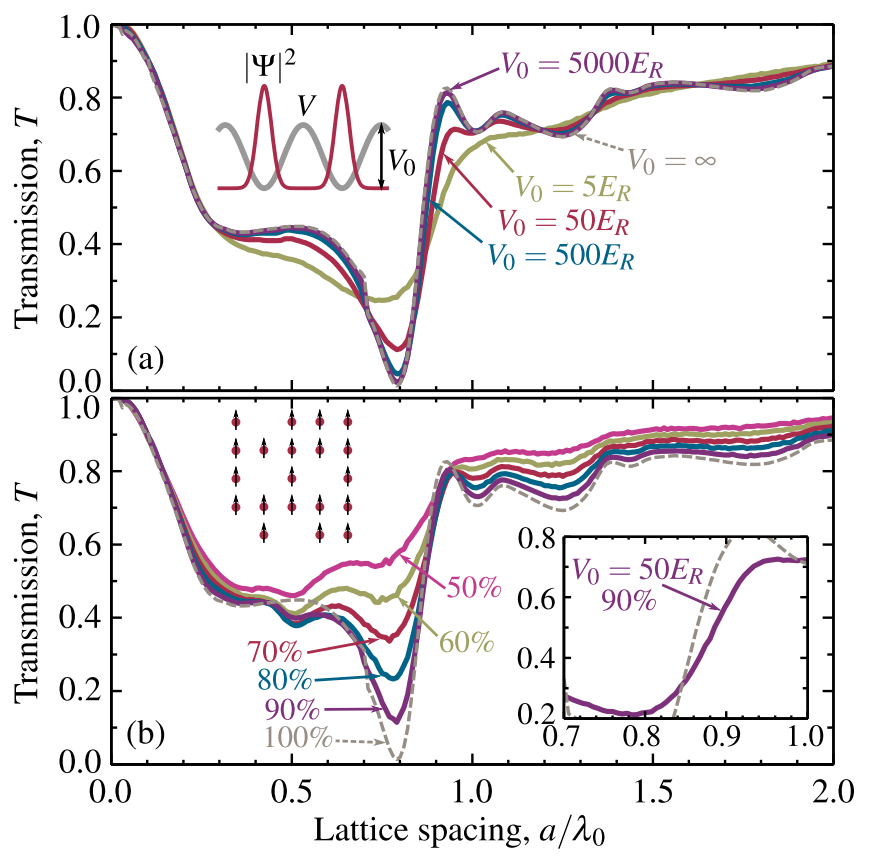

FIG. 4. The effect of finite trap depth (a) and finite filling factors (b) on resonant optical transmission through a $10 \times 10$ square lattice. (a) The trap depths are $V_{0}=\infty$ (grey dashed), $V_{0}=5000 E_{R}$ (purple), $V_{0}=500 E_{R}$ (blue), $V_{0}=50 E_{R}$ (red), and $V_{0}=5 E_{R}$ (green), where $E_{R}$ is the recoil energy and the filling is $100 \%$. (b) The lattice sites are randomly occupied with filling factors of $100 \%$ (grey dashed), $90 \%$ (purple), $80 \%$ (blue), $70 \%$ (red), $60 \%$ (green), and $50 \%$ (pink), with $V_{0}=\infty$. The purple line in the inset is a combination of finite trap depth $\left(V_{0}=50 E_{R}\right)$ and $90 \%$ filling. Each line is an average of several hundred realizations. The same lens and beam parameters as in Fig. 1 are used.

hexagonal $\left(\epsilon>98 \%\right.$ at $a=0.6 \lambda_{0}$ ) lattices with $N \sim 100$, providing further choice of trapping geometry. The complexity of the long-range many body coupling responsible for this behavior means an analytic treatment is beyond the scope of this Letter. We do, however, observe trends; for example, the position of the magic lattice spacing increases with packing efficiency $\left(a / \lambda_{0}=\{0.6,0.79,0.87\}\right.$ for hexagonal, square, and triangular lattices, respectively).

When considering a possible realization of this in an atomic experiment, it is necessary to consider how effects such as finite trapping depth [Fig. 4(a)] and imperfect filling [Fig. 4(b)] affect the extinction. We model finite trapping depth $V_{0}$ by treating each atomic wave function as a ground-state harmonic oscillator [31,52]. Averaging hundreds of realizations, atomic positions are sampled as Gaussian random variables centered on each lattice site with standard deviation related to $V_{0}$. Typical trap depths in Mott-insulator experiments lie in the range $V_{0}=$ $(20-50) E_{R}$ [35,53-55] ( $E_{R}$ is the recoil energy [31]), although $V_{0} \sim 10^{3} E_{R}$ is possible [53,54]. Filling efficiency greater than $90 \%$ can be achieved [54-57], which when combined with a trap depth of $V_{0}=50 E_{R}$ [Fig. 4(b), inset], still gives a significant range in transmission $[(21 \pm 5) \%$ to
$(72 \pm 2) \%$ between $a \simeq 0.8 \lambda_{0}$ and $\left.a \simeq 0.95 \lambda_{0}\right]$. The extinction is also robust to small changes in the direction of incidence of the laser; rotating the incident laser $10^{\circ}$ from the normal of a $10 \times 10$ square lattice still produces a peak extinction of over $90 \%$.

The number of lattice sites does not have to be large to observe strong extinction; a $4 \times 4$ perfect square lattice peaks at $\epsilon=96 \%$ (for $w_{0}=\lambda$ ). With $100 \%$ filling, increasing the atom number increases the peak extinction. The optimal beamwidth for maximizing the extinction scales with $\sqrt{N}\left(w_{0} \simeq 2.5 \lambda[58]\right.$ optimizes the extinction for square and triangular lattices with $N \simeq 100$ ). However, for 50\% filling as in Fig. 3(b), adding more lattice sites (e.g., 200 sites with 100 vacancies) makes little difference to the transmission, meaning high filling factors are essential for high extinction.

In conclusion, we have demonstrated numerically how the strong cooperative response of a $2 \mathrm{D}$ lattice of interacting dipoles can allow for very high extinctions (close to $100 \%$ ) without the need for high densities, large atom numbers, or strong focusing. The cavitylike dependence on spacing between atoms in these periodic lattices results in a strong dependence on the lattice spacing. Thanks to its efficient packing, the triangular lattice performs best, with a highly tunable transmission of between $<1 \%$ and $90 \%$ for a small change in lattice spacing. This work demonstrates further that the presence of interactions significantly modifies the optical response of a medium. Building on previous works in random gases $[18,20,21,45]$, we have shown that adding structure to the atom positions can significantly enhance such effects. By combining with Rydberg blockade, one could realize a dipolar QED analogue of the strong coupling regime in cavity QED, with potential applications for quantum nonlinear optics.

The data presented in this Letter can be found in Ref. [59].

We thank M. Greiner, C. Genes, H. Ritsch, S. Krämer, J. Ruostekoski, and R. Kaiser for helpful discussions. We acknowledge funding from the UK EPSRC (Grant No. EP/ L023024/1).

*r.j.bettles@durham.ac.uk

s.a.gardiner@durham.ac.uk

c.s.adams@durham.ac.uk

[1] G. Wrigge, I. Gerhardt, J. Hwang, G. Zumofen, and V. Sandoghdar, Nat. Phys. 4, 60 (2008).

[2] M. K. Tey, G. Maslennikov, T. C. H. Liew, S. A. Aljunid, F. Huber, B. Chng, Z. Chen, V. Scarani, and C. Kurtsiefer, New J. Phys. 11, 043011 (2009).

[3] Single dipole extinctions of $22 \%$ were demonstrated for molecules by selecting only the coherent dipolar emission [1].

[4] N. Piro, F. Rohde, C. Schuck, M. Almendros, J. Huwer, J. Ghosh, A. Haase, M. Hennrich, F. Dubin, and J. Eschner, Nat. Phys. 7, 17 (2011). 
[5] A. N. Vamivakas, M. Atatüre, J. Dreiser, S. T. Yilmaz, A. Badolato, A. K. Swan, B. B. Goldberg, A. Imamoğlu, and M. S. Ünlü, Nano Lett. 7, 2892 (2007).

[6] M. Mücke, E. Figueroa, J. Bochmann, C. Hahn, K. Murr, S. Ritter, C. J. Villas-Boas, and G. Rempe, Nature (London) 465, 755 (2010).

[7] J. D. Thompson, T. G. Tiecke, N. P. de Leon, J. Feist, A. V. Akimov, M. Gullans, A. S. Zibrov, V. Vuletić, and M. D. Lukin, Science 340, 1202 (2013).

[8] R. H. Dicke, Phys. Rev. 93, 99 (1954).

[9] R. G. DeVoe and R. G. Brewer, Phys. Rev. Lett. 76, 2049 (1996).

[10] M. Scheibner, T. Schmidt, L. Worschech, A. Forchel, G. Bacher, T. Passow, and D. Hommel, Nat. Phys. 3, 106 (2007).

[11] R. Röhlsberger, K. Schlage, B. Sahoo, S. Couet, and R. Rüffer, Science 328, 1248 (2010).

[12] A. Goban, C.-L. Hung, J. D. Hood, S.-P. Yu, J. A. Muniz, O. Painter, and H. J. Kimble, Phys. Rev. Lett. 115, 063601 (2015).

[13] J. Keaveney, A. Sargsyan, U. Krohn, I. G. Hughes, D. Sarkisyan, and C. S. Adams, Phys. Rev. Lett. 108, 173601 (2012).

[14] Z. Meir, O. Schwartz, E. Shahmoon, D. Oron, and R. Ozeri, Phys. Rev. Lett. 113, 193002 (2014).

[15] R. J. Bettles, S. A. Gardiner, and C. S. Adams, Phys. Rev. A 92, 063822 (2015).

[16] B. Luk'yanchuk, N. I. Zheludev, S. A. Maier, N. J. Halas, P. Nordlander, H. Giessen, and C. T. Chong, Nat. Mater. 9, 707 (2010).

[17] S. D. Jenkins and J. Ruostekoski, Phys. Rev. Lett. 111, 147401 (2013).

[18] J. Keaveney, A. Sargsyan, U. Krohn, J. Gontcharov, I. G. Hughes, D. Sarkisyan, and C. S. Adams, arXiv:1109.3669.

[19] S. P. Rath, T. Yefsah, K. J. Günter, M. Cheneau, R. Desbuquois, M. Holzmann, W. Krauth, and J. Dalibard, Phys. Rev. A 82, 013609 (2010).

[20] J. Pellegrino, R. Bourgain, S. Jennewein, Y. R. P. Sortais, A. Browaeys, S. D. Jenkins, and J. Ruostekoski, Phys. Rev. Lett. 113, 133602 (2014).

[21] K. Kemp, S. J. Roof, M. D. Havey, I. M. Sokolov, and D. V. Kupriyanov, arXiv:1410.2497.

[22] P. Ghenuche, G. Vincent, M. Laroche, N. Bardou, R. Haïdar, J.-L. Pelouard, and S. Collin, Phys. Rev. Lett. 109, 143903 (2012).

[23] V. A. Fedotov, N. Papasimakis, E. Plum, A. Bitzer, M. Walther, P. Kuo, D. P. Tsai, and N. I. Zheludev, Phys. Rev. Lett. 104, 223901 (2010).

[24] T. W. Ebbesen, H. J. Lezec, H. F. Ghaemi, T. Thio, and P. A. Wolff, Nature (London) 391, 667 (1998).

[25] S. D. Jenkins and J. Ruostekoski, New J. Phys. 14, 103003 (2012).

[26] L. Martín-Moreno, F. J. García-Vidal, H. J. Lezec, K. M. Pellerin, T. Thio, J. B. Pendry, and T. W. Ebbesen, Phys. Rev. Lett. 86, 1114 (2001).

[27] F. J. García de Abajo, Rev. Mod. Phys. 79, 1267 (2007).

[28] S. Krämer, L. Ostermann, and H. Ritsch, arXiv:1506.09079.

[29] M. Antezza and Y. Castin, Phys. Rev. A 80, 013816 (2009).

[30] M. Antezza and Y. Castin, Phys. Rev. A 88, 033844 (2013).
[31] See the Supplemental Material at http://link.aps.org/ supplemental/10.1103/PhysRevLett.116.103602, which includes Refs. [32,33], for details of the electric fields, choice of parameters and optical trapping.

[32] J. D. Jackson, Classical Electrodynamics (Wiley \& Sons, New York, 1963).

[33] S. J. van Enk and H. J. Kimble, Phys. Rev. A 63, 023809 (2001).

[34] B. Olmos, D. Yu, Y. Singh, F. Schreck, K. Bongs, and I. Lesanovsky, Phys. Rev. Lett. 110, 143602 (2013).

[35] T. Fukuhara, S. Sugawa, M. Sugimoto, S. Taie, and Y. Takahashi, Phys. Rev. A 79, 041604 (2009).

[36] S. D. Jenkins and J. Ruostekoski, Phys. Rev. B 86, 085116 (2012).

[37] B. Hopkins, A. N. Poddubny, A. E. Miroshnichenko, and Y. S. Kivshar, Phys. Rev. A 88, 053819 (2013).

[38] A. A. Svidzinsky, J. T. Chang, and M. O. Scully, Phys. Rev. A 81, 053821 (2010).

[39] G. Zumofen, N. M. Mojarad, V. Sandoghdar, and M. Agio, Phys. Rev. Lett. 101, 180404 (2008).

[40] M. Fischer, M. Bader, R. Maiwald, A. Golla, M. Sondermann, and G. Leuchs, Appl. Phys. B 117, 797 (2014).

[41] S. A. Aljunid, G. Maslennikov, Y. Wang, H. L. Dao, V. Scarani, and C. Kurtsiefer, Phys. Rev. Lett. 111, 103001 (2013).

[42] D. Paredes-Barato and C. S. Adams, Phys. Rev. Lett. 112, 040501 (2014).

[43] R. Friedberg, S. R. Hartmann, and J. T. Manassah, Phys. Rep. 7, 101 (1973).

[44] J. Javanainen, J. Ruostekoski, Y. Li, and S. M. Yoo, Phys. Rev. Lett. 112, 113603 (2014).

[45] L. Chomaz, L. Corman, T. Yefsah, R. Desbuquois, and J. Dalibard, New J. Phys. 14, 055001 (2012).

[46] J. Ruostekoski and J. Javanainen, Phys. Rev. A 55, 513 (1997).

[47] J. Javanainen, J. Ruostekoski, B. Vestergaard, and M. R. Francis, Phys. Rev. A 59, 649 (1999).

[48] M. Greiner, O. Mandel, T. Esslinger, T. W. Hänsch, and I. Bloch, Nature (London) 415, 39 (2002).

[49] P. Windpassinger and K. Sengstock, Rep. Prog. Phys. 76, 086401 (2013).

[50] F. Nogrette, H. Labuhn, S. Ravets, D. Barredo, L. Béguin, A. Vernier, T. Lahaye, and A. Browaeys, Phys. Rev. X 4, 021034 (2014).

[51] J.-P. Hugonin, M. Besbes, and P. Ben-Abdallah, Phys. Rev. B 91, 180202 (2015).

[52] S. D. Jenkins and J. Ruostekoski, Phys. Rev. A 86, 031602 (2012).

[53] W. S. Bakr, J. I. Gillen, A. Peng, S. Fölling, and M. Greiner, Nature (London) 462, 74 (2009).

[54] J. F. Sherson, C. Weitenberg, M. Endres, M. Cheneau, I. Bloch, and S. Kuhr, Nature (London) 467, 68 (2010).

[55] W. S. Bakr, P. M. Preiss, M. E. Tai, R. Ma, J. Simon, and M. Greiner, Nature (London) 480, 500 (2011).

[56] W. S. Bakr, A. Peng, M. E. Tai, R. Ma, J. Simon, J. I. Gillen, S. Folling, L. Pollet, and M. Greiner, Science 329, 547 (2010).

[57] B. J. Lester, N. Luick, A. M. Kaufman, C. M. Reynolds, and C. A. Regal, Phys. Rev. Lett. 115, 073003 (2015).

[58] The vector field propagation model results in a beam waist of $w_{0} \simeq 2.52 \lambda[31]$.

[59] DOI: $10.15128 / \mathrm{vt} 150 \mathrm{j} 378$. 